\title{
New Master Mapping Reference Table (MMRT) to Assist ICD-10 Transition for Syndromic Surveillance
}

\author{
Brooke Evans ${ }^{* 1}$, Peter Hicks ${ }^{3}$, Julie A. Pavlin ${ }^{4}$, Aaron Kite-Powell7, Atar Baer ${ }^{5}$, David J. \\ Swenson $^{6}$, Rebecca Lampkins ${ }^{2}$, Achala U. Jayatilleke ${ }^{3}$ and Laura Streichert ${ }^{1}$
}

${ }^{1}$ ISDS, Boston, MA, USA; ${ }^{2} \mathrm{CSTE}$, Atlanta, GA, USA; ${ }^{3} \mathrm{CDC}$, Atlanta, GA, USA; ${ }^{4}$ Infectious Disease Clinical Research Program \& HJF, USUHS, Bethesda, MD, USA; ${ }^{P}$ Public Health - Seattle \& King County, Seattle, WA, USA; ${ }^{6}$ State of New Hampshire, Concord, NH, USA $;{ }^{7}$ Armed Forces Health Surveillance Center, Silver Spring, MD, USA

\section{Objective}

To describe the process undertaken to translate syndromic surveillance syndromes and sub-syndromes consisting of ICD-9 $\mathrm{CM}$ diagnostic codes to syndromes and sub-syndromes consisting of ICD-10-CM codes, and how these translations can be used to improve syndromic surveillance practice.

\section{Introduction}

As of October 1, 2015, all HIPAA covered entities transition from the use of International Classification of Diseases version 9 (ICD-9-CM) to version 10 (ICD-10-CM/PCS). Many Public Health surveillance entities receive, interpret, analyze, and report ICD-9 encoded data, which will all be significantly impacted by the transition. Public health agencies will need to modify existing database structures, extraction rules, and messaging guides, as well as revise established syndromic surveillance definitions and underlying analytic and business rules to accommodate this transition. Implementation challenges include resource, funding, and time constraints for code translation and syndrome classification, and developing statistical methodologies to accommodate changes to coding practices.

To address these challenges, the International Society for Disease Surveillance (ISDS), in consultation with the Centers for Disease Control and Prevention (CDC) and the Council of State and Territorial Epidemiologists (CSTE), has conducted a project to develop consensus-driven syndrome definitions based on ICD$10-\mathrm{CM}$ codes. The goal was to have the newly created ICD-9-CMto-ICD-10-CM mappings and corresponding syndromic definitions fully reviewed and vetted by the syndromic surveillance community, which relies on these codes for routine surveillance, as well as for research purposes. The mappings may be leveraged by other federal, state, and local public health entities to better prepare and improve the surveillance, analytics, and reporting activities impacted by the ICD-10-CM transition.

\section{Methods}

ISDS coordinated a multi-stakeholder working group to revisit existing syndromic surveillance definitions and compile ICD-9-CM codes that originated in BioSense that map to these categories. The individual ICD-9-CM codes within each category were then mapped to the 2013 ICD-10-CM using General Equivalence Mappings (GEMs). Subsequently, we followed a reverse translation validation process to ensure that the appropriate codes were correctly identified. The resulting Master Mapping Reference Table (MMRT) relates syndromic classifications to both code groupings. The code mappings were then reviewed by the surveillance community and partner agencies, leveraging clinical and epidemiological expertise, to reach consensus.

\section{Results}

The new MMRT tool, released in August 2015, provides a key resource to public health practitioners that use syndromic surveillance to update their systems and to correctly identify trends over time that span the transition period by using both code sets, as well as surveillance activities using exclusively ICD-10 CM.

\section{Conclusions}

The development of a consensus-driven MMRT assists entities with the complex task of translating ICD-9-CM to ICD-10-CM codes. It is anticipated that the higher level of detail inherent to ICD10-CM codes will improve the specificity of syndromic surveillance. The code translations will also serve to develop standardized syndrome definitions based on conceptual mappings and a deductive development approach from concept to diagnostic codes to syndromes.

Finally, the mappings will enable users to address challenges associated with changes in baseline trends (Figure 1) as a result of the transition. Leveraging the MMRT, jurisdictions can quickly map forwards and backwards across the two coding systems to ensure continuity of analytics and reporting during the transition period.

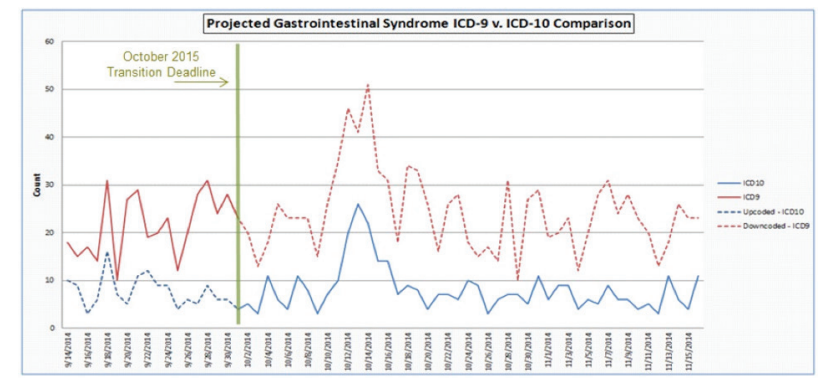

\section{Keywords}

ICD-10; syndrome definitions; syndromic surveillance; codemapping; ICD-10 transition

\section{Acknowledgments}

We thank the surveillance professionals that assisted with the code set review. This work was supported by the CDC.

\section{*Brooke Evans}

E-mail: bevans@syndromic.org 